\title{
Optical Signatures of Periodic Charge Distribution in a Mott-like Correlated Insulator State
}

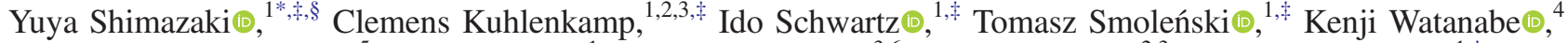 \\ Takashi Taniguchi, ${ }^{5}$ Martin Kroner, ${ }^{1}$ Richard Schmidt $\odot,{ }^{3,6}$ Michael Knap $\odot,{ }^{2,3}$ and Ataç Imamoğlu ${ }^{1, \dagger}$ \\ ${ }^{1}$ Institute for Quantum Electronics, ETH Zürich, CH-8093 Zürich, Switzerland \\ ${ }^{2}$ Department of Physics and Institute for Advanced Study, Technical University of Munich, \\ 85748 Garching, Germany \\ ${ }^{3}$ München Center for Quantum Science and Technology, Schellingstrasse 4, 80799 Münich, Germany \\ ${ }^{4}$ Research Center for Functional Materials, National Institute for Materials Science, \\ Tsukuba, Ibaraki 305-0044, Japan \\ ${ }^{5}$ International Center for Materials Nanoarchitectonics, National Institute for Materials Science, \\ Tsukuba, Ibaraki 305-0044, Japan \\ ${ }^{6}$ Max Planck Institute of Quantum Optics, 85748 Garching, Germany
}

(Received 6 October 2020; revised 19 January 2021; accepted 15 March 2021; published 4 May 2021)

The elementary optical excitations in two-dimensional semiconductors hosting itinerant electrons are attractive and repulsive polarons-excitons that are dynamically screened by electrons. Exciton polarons have hitherto been studied in translationally invariant degenerate Fermi systems. Here, we show that periodic distribution of electrons breaks the excitonic translational invariance and leads to a direct optical signature in the exciton-polaron spectrum. Specifically, we demonstrate that new optical resonances appear due to spatially modulated interactions between excitons and electrons in an incompressible Mott-like correlated state. Our observations demonstrate that resonant optical spectroscopy provides an invaluable tool for studying strongly correlated states, such as Wigner crystals and density waves, where exciton-electron interactions are modified by the emergence of charge order.

DOI: 10.1103/PhysRevX.11.021027

\section{INTRODUCTION}

Bilayer graphene and transition metal dichalcogenide (TMD) heterostructures have emerged as fascinating new platforms to realize and probe exotic phases of quantum matter. Introducing a twist angle or lattice constant mismatch between the two layers allows for combining a moiré superlattice and long-range Coulomb interactions, which, in turn, leads to the formation of strongly correlated electronic states [1-3]. In contrast to twisted bilayer graphene, TMD-based heterostructures allow for the use of resonant optical reflection spectroscopy to study the signatures of new electronic phases, such as correlated

*yuya.shimazaki@riken.jp

†imamoglu@phys.ethz.ch

These authors contributed equally to this work.

${ }^{\S}$ Present address: Center for Emergent Matter Science, RIKEN, 2-1 Hirosawa, Wako, Saitama 351-0198, Japan.

Published by the American Physical Society under the terms of the Creative Commons Attribution 4.0 International license. Further distribution of this work must maintain attribution to the author(s) and the published article's title, journal citation, and DOI.
Subject Areas: Condensed Matter Physics,

Semiconductor Physics,

Strongly Correlated Materials

insulator states at fractional fillings that break discrete translational symmetry, in van der Waals heterostructures [4-7]. While these discoveries have sparked the development of several novel methods to characterize such states [8], most of the reported signatures are not directly sensitive to the spatial correlations of the quantum state of electrons.

Here, we show a striking, yet generic optical signature of the emergence of periodic ordering of electrons in a lattice structure. Because of strong exciton-electron interactions characteristic of TMD monolayers [9-11], the formation of a Mott or Wigner state of electrons [12] creates a periodic potential for excitons, thereby modifying the excitonpolaron $[13,14]$ spectrum. Our experiments show that when either or both of the layers host an electronic Mott-like correlated insulator (CI) state, a new umklapp excitonpolaron peak appears in the resonant reflection spectrum. The energy shift of this new resonance is determined by the lattice constant of the emerging Mott-like CI state and by the strength of exciton-electron interaction.

In our experiments, we study a twisted $\mathrm{MoSe}_{2} / \mathrm{hBN} /$ $\mathrm{MoSe}_{2}$ homobilayer structure, which exhibits an incompressible single-layer Mott-like CI state for unity filling of the underlying electronic moiré potential [6]. In comparison to heterobilayer structures [4,5], the presence of 
(a)

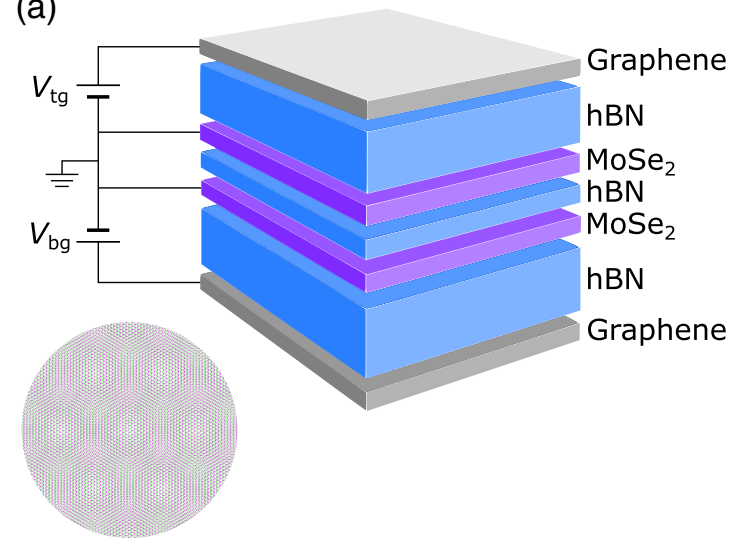

(c)

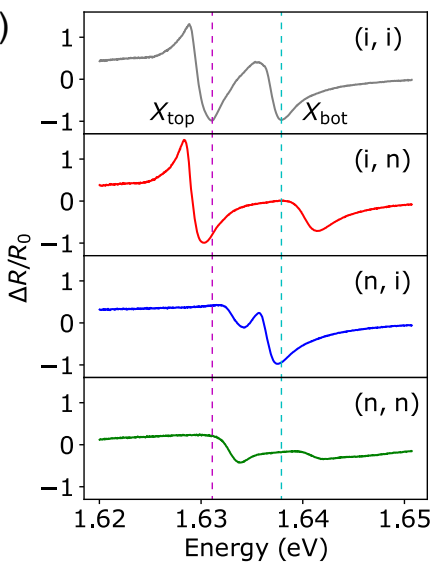

(b)

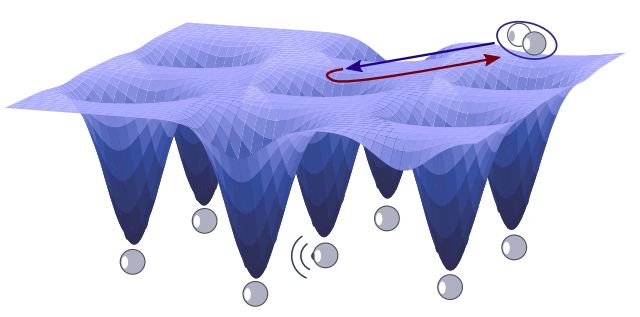

Electron (d)

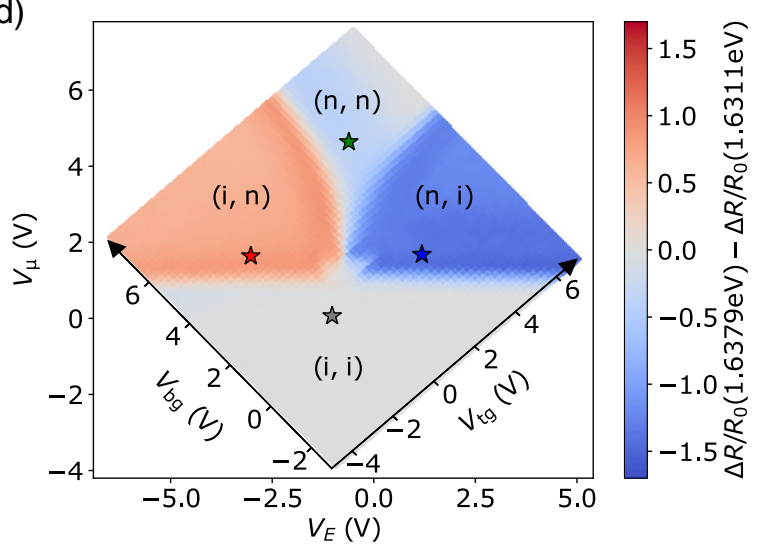

FIG. 1. Moiré lattice in $\mathrm{MoSe}_{2} / \mathrm{hBN} / \mathrm{MoSe}_{2}$ and basic characterization. (a) Schematic picture of the device structure. The left bottom picture shows a moiré lattice. (b) Schematic picture of the potential for excitons created by electrons trapped in a moiré lattice. (c) Differential reflectance spectrum for each charge configuration. "i" and "n" indicate neutral and electron doped charge configurations and are shown in the order of (top, bottom). (d) Color map of layer contrasted differential reflectance signal $\left[\Delta R / R_{0}(1.6379 \mathrm{eV})-\Delta R / R_{0}(1.6311 \mathrm{eV})\right]$.

monolayer hBN in between the $\mathrm{MoSe}_{2}$ monolayers leads to two new features: First, the on-site and possibly intersite Coulomb repulsion energy exceeds the strength of the moiré potential, which is drastically weakened by the hBN monolayer. Second, the energy difference between the electronic states in the two layers is tunable, resulting in a robust layer pseudospin degree of freedom that can be controlled using an applied vertical electric field.

Before proceeding, we note that recent photoluminescence experiments allow for the characterization of a static excitonic moiré potential in twisted heterobilayers [1519]. This periodic potential is particularly strong for interlayer excitons [20-22]: In structures where two different TMD monolayers are in direct contact, new interand intralayer excitonic resonances arising either from localization at high-symmetry stacking points or from umklapp processes are observed. In stark contrast to these earlier works, the periodic static moiré potential experienced by the excitons in our sample is weak as compared to the exciton linewidth due to the $\mathrm{hBN}$ barrier layer: As a consequence, we observe only a single intralayer exciton resonance in the absence of electron or hole doping.

\section{BASIC CHARACTERIZATION}

Figure 1(a) shows the sample we study in our experiments: Two $\mathrm{MoSe}_{2}$ layers are isolated by monolayer hBN and are encapsulated between two thick hBN layers. Fewlayer graphene sheets on the top and bottom of the device are used as transparent gate electrodes; we apply top and bottom gate voltages ( $V_{\mathrm{tg}}$ and $V_{\mathrm{bg}}$, respectively) while keeping the $\mathrm{MoSe}_{2}$ layers grounded. A small twist angle between the top and bottom $\mathrm{MoSe}_{2}$ layers results in the formation of a moiré superlattice, as shown schematically in the left bottom part in Fig. 1(a). In our previous work [6] based on the same device, we estimate the twist angle to be around $0.8^{\circ}$, which corresponds to the moiré lattice constant $a_{M} \sim 25 \mathrm{~nm}$ (see also Supplemental Material S2 [23]). In the limit where each moiré unit cell of a single layer is singly occupied, the trapped electrons form a triangular lattice [Fig. 1(b)]. We emphasize that the moiré potential between $\mathrm{hBN}$ and $\mathrm{MoSe}_{2}$ is negligible. The reason is that the lattice constants of $\mathrm{hBN}$ and $\mathrm{MoSe}_{2}$ are largely mismatched $\left(a_{\mathrm{hBN}}=2.51 \AA\right.$ [24] and $a_{\mathrm{MoSe}_{2}}=$ $3.32 \AA[25])$. Moreover, neither the monolayer hBN spacer, 
(a)

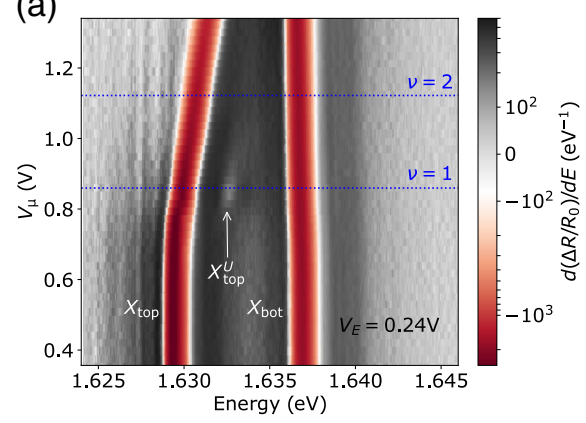

(b)

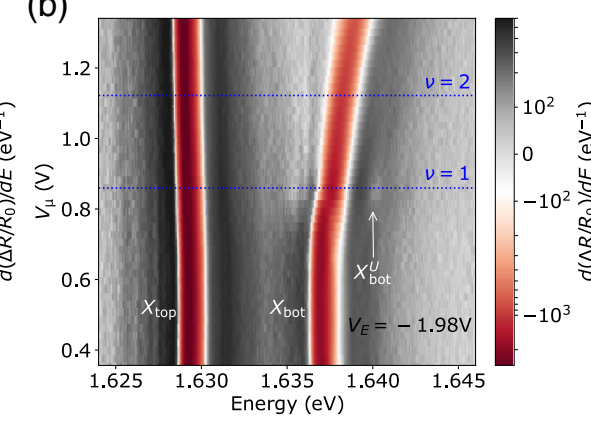

(c)

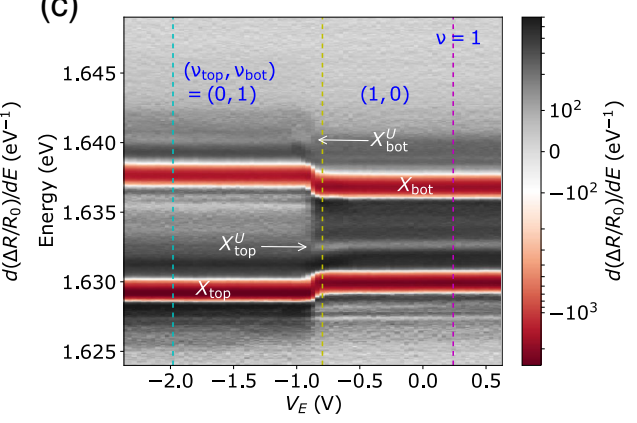

FIG. 2. Umklapp exciton resonances at $\nu=1$. (a),(b) $V_{\mu}$ dependence of differential reflectance differentiated with respect to energy $E$ at $V_{E}=0.24 \mathrm{~V}$ (a) and $V_{E}=-1.98 \mathrm{~V}$ (b). (c) $V_{E}$ dependence of differential reflectance spectra at $\nu=1$. The cyan, yellow, and magenta dashed lines correspond to $V_{E}=-1.98,-0.80$, and $0.24 \mathrm{~V}$, respectively. The scale of the color bars is logarithmic for $\left|d\left(\Delta R / R_{0}\right) / d E\right|>10^{2} \mathrm{eV}^{-1}$ and linear for $\left|d\left(\Delta R / R_{0}\right) / d E\right|<10^{2} \mathrm{eV}^{-1}$.

nor the top hBN layer, nor the bottom $\mathrm{hBN}$ layer are aligned to the $\mathrm{MoSe}_{2}$ layers.

Figure $1(\mathrm{c})$ shows the differential reflectance $\left[\Delta R / R_{0}=\right.$ $\left(R-R_{0}\right) / R_{0}$ ] spectrum around the exciton resonances for different charge configurations of the two $\mathrm{MoSe}_{2}$ layers. Here, $R$ denotes the reflectance of the $\mathrm{MoSe}_{2} / \mathrm{hBN} / \mathrm{MoSe}_{2}$ homobilayer structure, whereas $R_{0}$ is the background reflectance obtained from a region of the sample without $\mathrm{MoSe}_{2}$ layers. All experiments depicted in the main text are performed using a cryogenic confocal microscope setup operated at a temperature of approximately $4 \mathrm{~K}$. The results we present in Secs. II and III are obtained in the absence of an external magnetic field $\left(B_{z}=0\right)$. The gray curve in the upper panel depicts $\Delta R / R_{0}$ when both layers are in the charge-neutral regime. Accordingly, the two resonances correspond to the top and bottom layer excitons ( $X_{\text {top }}$ and $X_{\text {bot }}$, respectively) whose energies differ by approximately $8 \mathrm{meV}$ due to inhomogeneous strain [26-28]. When one of the layers is electron doped, exciton-electron interactions lead to a blueshift of the repulsive polaron resonance frequency $[13,14]$. Since the exciton-electron interactions are short ranged, excitons in a given layer scatter predominantly with electrons in the same layer. By monitoring the magnitude of $\Delta R / R_{0}$ at a fixed energy close to either $X_{\text {top }}$ or $X_{\text {bot }}$ resonances, we can monitor the charge configuration of each layer. Since we do not consider the changes in the attractive polaron sector of the spectrum, we simply refer to repulsive polaron transitions as excitonic resonances in the following discussion.

Figure 1(d) shows the gate voltage dependence of the layer contrasted $\Delta R / R_{0}$ signal, revealing the charge configuration of the device [6]. The color-coded plot shows the difference of $\Delta R / R_{0}$ signals at 1.6379 and $1.6311 \mathrm{eV}$ which are indicated with cyan and magenta lines in Fig. 1(c), respectively. We define the gate voltage axes as $V_{E}=0.5 V_{\mathrm{tg}}-0.5 V_{\mathrm{bg}}$ and $V_{\mu}=0.4561 V_{\mathrm{tg}}+0.5439 V_{\mathrm{bg}}$, which, respectively, correspond to changes of the top and bottom gate voltages that leave the chemical potential and the electric field invariant in the charge-neutral regime.
The combinations of $V_{E}$ and $V_{\mu}$ used to obtain the spectra in Fig. 1(c) are marked with color-coded stars in Fig. 1(d).

\section{SPECTROSCOPIC SIGNATURE OF EXCITON UMKLAPP SCATTERING IN MOTT-LIKE STATES}

Strong electronic correlations become manifest in a half-filled moiré subband $(\nu=1)$, where each moiré site is occupied by a single electron. Since our structure has a layer degree of freedom, we specify the electron filling factor of top and bottom layers as $\nu_{\text {top }}$ and $\nu_{\text {bot }}$, respectively, with $\nu=\nu_{\text {top }}+\nu_{\text {bot }}$ (see Supplemental Material S2 [23] for the identification of filling). In Fig. 2(a), we show $V_{\mu}$ dependence of reflectance spectrum at a fixed $V_{E}=0.24 \mathrm{~V}$. To better visualize small signals, we take a moving average of $\Delta R / R_{0}$ within a $0.24 \mathrm{meV}$ energy window and plot the derivative of $\Delta R / R_{0}$ with respect to energy. The bottom layer remains neutral at this $V_{E}$, and only the filling of the top layer is modulated along the $V_{\mu}$ axis, as evidenced by the blueshift of $X_{\text {top }}$ resonance. Remarkably, we observe that, once the top layer is doped to $\nu=1$ where $\left(\nu_{\text {top }}, \nu_{\text {bot }}\right)=(1,0)$, an additional higherenergy exciton resonance labeled as $X_{\text {top }}^{U}$ appears. The estimated energy separation between $X_{\text {top }}$ and $X_{\text {top }}^{U}$, determined from resonant fluorescence data, is $\simeq 2.7 \mathrm{meV}$ (see Supplemental Material S1 [23]).

The corresponding resonance also appears when the bottom layer is doped to $\nu=1$ : Figure 2(b) shows the $V_{\mu}$ dependence of $d\left(\Delta R / R_{0}\right) / d E$ at a fixed $V_{E}=-1.98 \mathrm{~V}$, where we fill only the bottom layer and the top layer remains charge neutral. At $\nu=1$ where $\left(\nu_{\text {top }}, \nu_{\text {bot }}\right)=(0,1)$, we also observe a faint resonance labeled as $X_{\text {bot }}^{U}$ on the blue side of $X_{\text {bot }}$. The energy separation between $X_{\text {bot }}$ and $X_{\text {bot }}^{U}$ is also estimated to be $\simeq 2.7 \mathrm{meV}$.

Figure 2(c) shows the $V_{E}$ dependence of $d\left(\Delta R / R_{0}\right) / d E$ at $\nu=1$. The sharp energy shift of the $X_{\text {top }}$ and $X_{\text {bot }}$ resonances around $V_{E} \sim-0.9 \mathrm{~V}$ signals an abrupt transfer 
of all electrons from one layer to another upon minute changes in the electric field $E_{z}$. As $\nu$ is increased or decreased away from 1 (one electron per moiré site), the electrons are transferred from one layer to another gradually (see Fig. S3 in Supplemental Material [23]). These observations indicate the formation of a Mott-like CI state at $\nu=1$ [6]: The abrupt transfer of electrons, in turn, indicates that the potential minima of the bottom and top layer moiré potentials for electrons are displaced from each other. By transferring the Mott-like insulating phase from the top $\left[\left(\nu_{\text {top }}, \nu_{\text {bot }}\right)=(1,0)\right]$ to the bottom $\left[\left(\nu_{\text {top }}, \nu_{\text {bot }}\right)=(0,1)\right]$ layer, we find the $X_{\text {top }}^{U}$ resonance disappears and $X_{\text {bot }}^{U}$ resonance appears. In contrast to strong $V_{\mu}$ dependence, the $X_{\text {top }}^{U}$ and $X_{\text {bot }}^{U}$ resonances consistently exist for a wide range of $V_{E}$ at $\nu=1$.

Since the new excitonic resonance in the top (bottom) layer appears exclusively when $\nu_{\text {top }}=1\left(\nu_{\text {bot }}=1\right)$, we explain its origin as the emergence of a periodic potential for excitons generated by electrons in the singly occupied Mott-like CI state. Exciton-electron interactions ensure scattering of resonantly generated excitons off this Mott-like-state potential and lead to the emergence of excitonic bands in the new reduced exciton Brillouin zone. In the limit of weak excitonelectron scattering, the new excitonic resonances can be understood as umklapp processes. In this limit, the energy of the umklapp scattered exciton is determined by the reciprocal lattice vector of the electron-induced potential alone, which is fixed by the moiré periodicity. The estimated lattice constant of $\simeq 25 \mathrm{~nm}$ for this sample [6] yields a splitting of $\simeq 2.5 \mathrm{meV}$, which is in very good agreement with the experimentally observed splitting of the umklapp resonance (see the Appendix B).

Before presenting a theoretical model for these experimental observations, we investigate the optical response when there are two electrons per moiré site $(\nu=2)$. Figure 3(a) shows $V_{\mu}$ dependence of $d\left(\Delta R / R_{0}\right) / d E$ at a fixed $V_{E}=-0.80 \mathrm{~V}$. At this $V_{E}$, indicated with a yellow dashed line in Fig. 2(c), the system is filled as $\left(\nu_{\text {top }}, \nu_{\text {bot }}\right)=$ $(1,0)$ at $\nu=1$ and $\left(\nu_{\text {top }}, \nu_{\text {bot }}\right)=(1,1)$ at $\nu=2$. Consistent with the measurements depicted in Fig. 2(a), we find the emergence of $X_{\text {top }}^{U}$ at $\nu=1$ in Fig. 3(a). Remarkably, at $\nu=2$ where $\left(\nu_{\text {top }}, \nu_{\text {bot }}\right)=(1,1)$, the umklapp exciton resonances $X_{\text {top }}^{U}$ and $X_{\text {bot }}^{U}$ emerge simultaneously. This observation at $\left(\nu_{\text {top }}, \nu_{\text {bot }}\right)=(1,1)$ unequivocally shows the essential role played by the CI state of electrons in effecting a periodic potential for excitons. The estimated energy separation between $X_{\text {top }}$ and $X_{\text {top }}^{U}$ is $\simeq 2.8 \mathrm{meV}$; similarly, the separation between $X_{\text {bot }}$ and $X_{\text {bot }}^{U}$ is $\simeq 2.6 \mathrm{meV}$. The magnitude of these energy separations is very similar to the ones obtained at $\nu=1$, indicating that the top and bottom layers have the same triangular electron lattices and that the periodicity is not affected by the
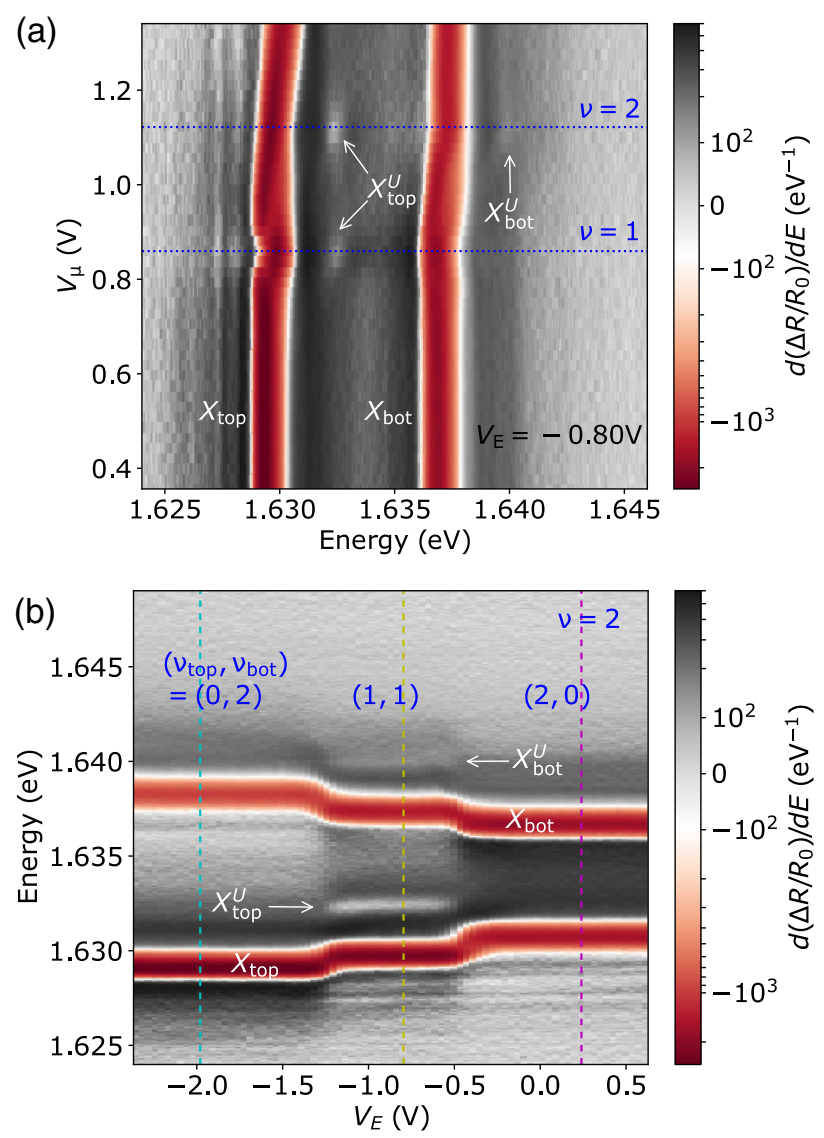

FIG. 3. Umklapp exciton resonances at $\nu=2$. (a) $V_{\mu}$ dependence of differential reflectance differentiated with respect to energy $E$ at $V_{E}=-0.80 \mathrm{~V}$. (b) $V_{E}$ dependence of differential reflectance spectra at $\nu=2$. The cyan, yellow, and magenta dashed lines correspond to $V_{E}=-1.98,-0.80$, and $0.24 \mathrm{~V}$, respectively. The scale of the color bars is logarithmic for $\left|d\left(\Delta R / R_{0}\right) / d E\right|>10^{2} \mathrm{eV}^{-1}$ and linear for $\left|d\left(\Delta R / R_{0}\right) / d E\right|<$ $10^{2} \mathrm{eV}^{-1}$.

simultaneous presence of CI states in the two layers. The similarity of energy separation for $\nu=1$ and $\nu=2$ also suggests that these new resonances are unlikely to originate from shakeup processes, since the excitation spectrum of a CI state is expected to be sensitive to the filling.

Finally, we show $V_{E}$ dependence of $d\left(\Delta R / R_{0}\right) / d E$ at $\nu=2$ in Fig. 3(b). The yellow dashed line indicates $V_{E}=-0.80 \mathrm{~V}$; the plateau structure of the excitonic resonance energies for $-1.2 \leq V_{E} \leq-0.60 \mathrm{~V}$ shows the resilience of the layer occupancy to the applied electric field, indicating the formation of CI states simultaneously in both layers at $\left(\nu_{\text {top }}, \nu_{\text {bot }}\right)=(1,1)$. Consistently, we observe top and bottom layer umklapp excitonic resonances throughout the range of $V_{E}$ for which $\left(\nu_{\text {top }}, \nu_{\text {bot }}\right)=(1,1)$. We also remark that the umklapp resonances are absent for $\left(\nu_{\text {top }}, \nu_{\text {bot }}\right)=(2,0),(0,2)$ [which we also confirm from Figs. 2(a) and 2(b)], indicating that the electrons do not form a CI state with two electrons per moiré potential site. 
(a)

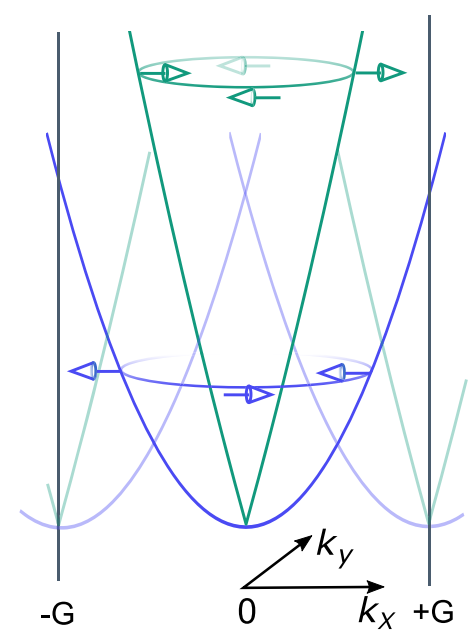

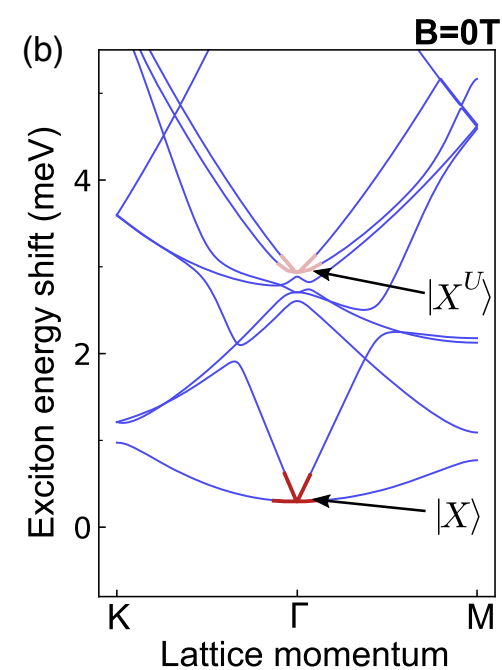

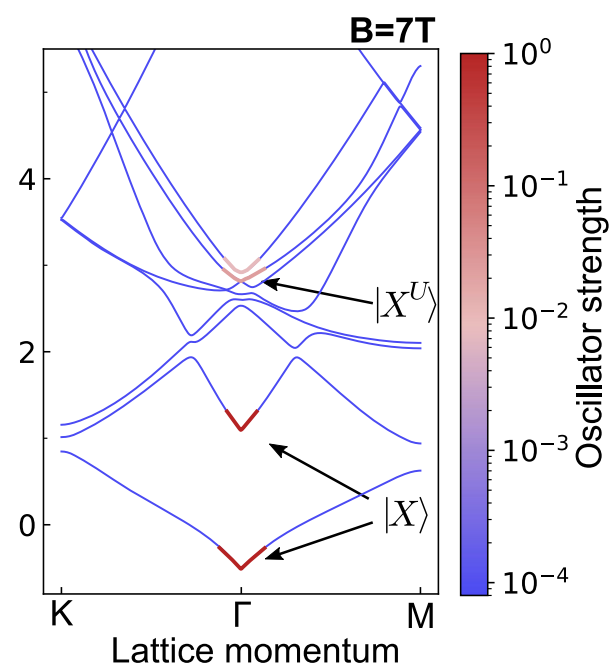

FIG. 4. Exciton band structure. Spectrum of the effective band Hamiltonian of Eq. (3) for parameters given in the main text and $J=300 \mathrm{meV}$. (a) Bare dispersion of mobile excitons. Strong intervalley electron-hole exchange coupling splits the linearly polarized exciton modes and yields two branches with linear (green line) and parabolic (blue line) dispersion. For simplicity, we do not show that the degeneracy of the excitonic branches extends throughout the light cone. The exciton valley pseudospin is shown as blue (green) arrows for the parabolic (linear) dispersion. Periodic potentials allow states connected by reciprocal lattice vectors to mix, yielding higher bands (transparent lines), as shown in an extended zone scheme. Higher bands of the linearly dispersing mode decouple due to their large energy detuning. (b) Exciton bands along a path in a moiré Brillouin zone. The oscillator strength of each state is indicated by the color bar, which is saturated for all blue lines. As photons carry almost vanishing momentum, only modes close to the $\Gamma$ point may obtain finite oscillator strength (we artificially extend the momentum range of the bright states for better visibility). While most states remain dark, only a single umklapp band per polarization obtains sizable oscillator strength. Left: dispersion in the absence of a magnetic field. We find an energy splitting between the umklapp state and the main resonance of 3 meV. This additional bright state carries $1.2 \%$ of the oscillator strength of the bare exciton. The oscillator strength of higher bands is significantly suppressed due to the larger energy splitting, which reduces the coupling to light. Right: exciton dispersion for $B_{z}=7 \mathrm{~T}$. While the main resonance splits significantly, the umklapp peaks are only marginally affected by the magnetic field.

\section{THEORETICAL MODEL}

The appearance of additional higher-energy excitonic resonances in the optical spectrum can be described by a simple model of excitons moving in a periodic potential. For simplicity, we are focusing only on a single layer which hosts the strongly correlated electronic state. As excitons are strongly bound, we consider them as mobile rigid quantum impurities. The two valleys of the TMD imprint as a (valley) pseudospin degree of freedom for the excitons. The optically active $k=0$ excitons are $\sigma^{+}$( $K$-valley) and $\sigma^{-}\left(K^{\prime}\right.$-valley) polarized and are degenerate. However, for exciton momenta outside the light cone, the long-range electron-hole exchange interaction strongly couples the $K$ and $K^{\prime}$ valley excitons $[29,30]$. In the pseudospin basis, where spin up (down) corresponds to an exciton in the $K\left(K^{\prime}\right)$ valley, the dynamics of an exciton is described by the following Hamiltonian:

$$
\begin{aligned}
H_{0}= & \sum_{\boldsymbol{k}}\left(\begin{array}{c}
x_{\boldsymbol{k}, K}^{\dagger} \\
x_{\boldsymbol{k}, K^{\prime}}^{\dagger}
\end{array}\right)^{T}\left[\frac{\hbar^{2} \boldsymbol{k}^{2}}{2 m_{X}}+\frac{|\boldsymbol{k}|}{|K|} J\left(\begin{array}{cc}
0 & e^{-i 2 \theta} \\
e^{+i 2 \theta} & 0
\end{array}\right)+\frac{|\boldsymbol{k}|}{|K|} J\right] \\
& \times\left(\begin{array}{c}
x_{k, K} \\
x_{\boldsymbol{k}, K^{\prime}}
\end{array}\right)
\end{aligned}
$$

where $x_{k, K}^{\dagger}\left(x_{k, K^{\prime}}^{\dagger}\right)$ creates an exciton in the $K\left(K^{\prime}\right)$ valley with center of mass momentum $\boldsymbol{k}, \hbar$ is Planck's constant, $m_{X}$ is the exciton mass, $\theta=\operatorname{atan}\left(k_{y} / k_{x}\right)$, and $J$ is the exchange coupling. The second and the third terms of Eq. (1) describe intervalley and intravalley exchange interaction, respectively. We remark that the exchange coupling is not easily accessible experimentally, and its exact value is, therefore, uncertain. While first-principle calculations yield large values of $J \sim 1 \mathrm{eV}$, we expect that the experimentally relevant coupling is likely to be reduced by dielectric screening due to the $\mathrm{hBN}$ encapsulation, as well as due to screening by electrons [31]. Our conclusions are, however, insensitive to the precise value of $J$ and are unaffected for a range of couplings $1 \mathrm{eV} \geq J \geq 150 \mathrm{meV}$. The exciton dispersions are given by $E(\boldsymbol{k})=\left(\hbar^{2} \boldsymbol{k}^{2} / 2 m_{X}\right)+(|\boldsymbol{k}| /|K|) J \pm(|\boldsymbol{k}| /|K|) J$ and are shown in Fig. 4(a). Exchange interactions split the polarizations into two branches with parabolic and linear dispersion. The linearly dispersing excitons fall on a steep cone, which leads to a large energy detuning from the parabolic branch.

The interaction between electrons and excitons is modeled by an effective repulsive contact interaction, which is justified, as we limit our discussion to features that appear 
at low energies $0 \leq E \leq 5 \mathrm{meV}$. The electron-exciton interaction Hamiltonian then takes the form

$$
H_{e-x}^{\mathrm{int}}=\int d^{2} r \lambda^{e-x}\left[\hat{n}_{e}(\boldsymbol{r}) \hat{n}_{X}^{K}(\boldsymbol{r})+\hat{n}_{e}(\boldsymbol{r}) \hat{n}_{X}^{K^{\prime}}(\boldsymbol{r})\right]
$$

where $\hat{n}_{X}^{K,\left(K^{\prime}\right)}$ is the density operator of excitons in the $K$ $\left(K^{\prime}\right)$ valley and $\hat{n}_{e}$ is the density operator of the electrons. We assume that the strength $\lambda^{e-x}$ of the interaction is the same for both polarizations and that the interaction itself is short range and repulsive. The delocalized nature of optically generated excitons ensures that the electronic Mott-like CI state, appearing due to an interplay between long-range Coulomb interactions and the moiré potential, is largely unperturbed by the exciton-electron interactions: This statement is verified, as the experimentally observed signatures of incompressible states are independent of the white-light intensity. To simplify the analysis, we assume that electrons are frozen in the Mott-like correlated ground state and describe the excitonic degrees of freedom alone by an effective Hamiltonian

$$
H_{\mathrm{eff}}=H_{0}+\int d^{2} r \lambda^{e-x}\left[\hat{n}_{X}^{K}(\boldsymbol{r})+\hat{n}_{X}^{K^{\prime}}(\boldsymbol{r})\right]\left\langle\hat{n}_{e}(\boldsymbol{r})\right\rangle,
$$

where $\left\langle\hat{n}_{e}(\boldsymbol{r})\right\rangle$ is the expectation value of the electron density. For the exciton mass, we use $m_{X}=m_{e}^{*}+m_{h}^{*}=$ $1.3 m_{e}$, where $m_{e}^{*}=0.7 m_{e}$ [32] and $m_{h}^{*}=0.6 m_{e}$ [33,34] are the effective masses of electrons and holes $\left(m_{e}\right.$ is the free electron mass). By using $H_{\text {eff }}$, we neglect the dynamical dressing of excitons by virtual excitations out of the CI state and take the effect of electronic correlations into account as a spatially dependent Hartree shift of the exciton energy, proportional to $\left\langle\hat{n}_{e}(\boldsymbol{r})\right\rangle$. While the precise shape of the potential seen by the excitons is determined by the electronic density and the moiré potential, we phenomenologically fix the Hartree shift induced by $\lambda^{e-x}$ to match the experimentally measured blueshift of the repulsivepolaron resonance induced by the electrons at low densities. We thereby determine $\lambda^{e-x}=2.1 \times 10^{-12} \mathrm{meV}$. $\mathrm{cm}^{2}$ to produce an excitonic blueshift of $0.4 \mathrm{meV}$ at fillings slightly away from $\nu=1$, where the electron density is expected to be homogeneous.

We solve the effective single-particle model of Eq. (3) assuming a Gaussian density profile for electrons:

$$
\left\langle\hat{n}_{e}(\boldsymbol{r})\right\rangle=\frac{1}{2 \pi \xi^{2}} \sum_{\boldsymbol{R}} e^{-\left(1 / 2 \xi^{2}\right)(\boldsymbol{r}-\boldsymbol{R})^{2}},
$$

where $\boldsymbol{R}$ is a triangular lattice vector and $\xi$ characterizes the extent of the electronic wave functions around the moiré sites [35]. We show the band structure resulting from the electron-induced potential in Fig. 4(b) for an electronic lattice with a lattice constant of $25 \mathrm{~nm}$ and $\xi=4 \mathrm{~nm}$. While the precise localization of the electrons is not known, we check that our results remain in reasonable agreement with the experiment for $\xi$ 's within $3 \mathrm{~nm}<\xi<6 \mathrm{~nm}$. The new bands in the moiré Brillouin zone appear as a consequence of the periodic excitonic potential, as momentum is no longer a good quantum number and is conserved only up to reciprocal lattice vectors $\mathbf{G}_{m}$. In particular, this result implies that excitons carrying a reciprocal lattice momentum $\boldsymbol{k}=\mathbf{G}_{m}$ now mix with the optically active $\boldsymbol{k}=0$ excitons via umklapp scattering.

The oscillator strength of the umklapp states is given by their zero-momentum exciton content. We numerically determine the relative oscillator strength of the first optically active umklapp band and find its relative oscillator strength to be

$$
\frac{\left|\left\langle\boldsymbol{k}=0 \mid X^{U}(\boldsymbol{\Gamma})\right\rangle\right|^{2}}{|\langle\boldsymbol{k}=0 \mid X(\boldsymbol{\Gamma})\rangle|^{2}} \simeq 1.2 \%,
$$

where $|X\rangle$ and $\left|X^{U}\right\rangle$ are the exciton and the bright umklappexciton states, respectively, of a given polarization, while $\Gamma$ labels vanishing lattice momentum. In Eq. (5), the vector $|\boldsymbol{k}\rangle$ is a plane wave state of the exciton with proper momentum $\boldsymbol{k}$. The amount of mixing and the band splittings are determined by the strength of the potential and the density profile of the electrons. However, the $C_{6}$ symmetry of the triangular potential restricts mixing with the $\boldsymbol{k}=0$ modes and, hence, the number of possible bright states: Only states which transform identically under rotations (and are, hence, grouped in the same $C_{6}$ representation) can have nonvanishing matrix elements. In our case, we find only two states among the first umklapp band with $C_{6}$ eigenvalues $\exp (i l \pi / 3)$, where $l= \pm 1$, which are circularly polarized and mix with the $|\boldsymbol{k}=0\rangle$ excitons to become bright. The oscillator strength of the bright resonances decreases rapidly as the umklapp energy increases; this feature renders only the first umklapp band effectively observable in experiments.

Both the appearance of a single umklapp line per polarization and its estimated oscillator strength are in good agreement with our experimental observations and confirm that umklapp exciton and repulsive-polaron resonances provide a direct probe of the periodic structure of the Mott-like CI state.

\section{MAGNETIC FIELD DEPENDENCE OF UMKLAPP STATES}

We now extend our analysis to finite magnetic fields $B_{z}$. As excitons are strongly bound and charge neutral, they couple only via an effective Zeeman term with a $g$ factor of approximately 4 [36-39]. We neglect the momentum dependence of the Zeeman shift and introduce it by adding a (momentum-independent) term $\hat{H}_{Z}=2 \mu_{B} B_{z} \sigma_{z}$ to the Hamiltonian of Eq. (3); here, $\mu_{B}$ is the Bohr magneton and $\sigma_{z}=\int d^{2} r \hat{n}_{X}^{K}(\boldsymbol{r})-\hat{n}_{X}^{K^{\prime}}(\boldsymbol{r})$. Since electron-hole exchange 
(a)

(a)

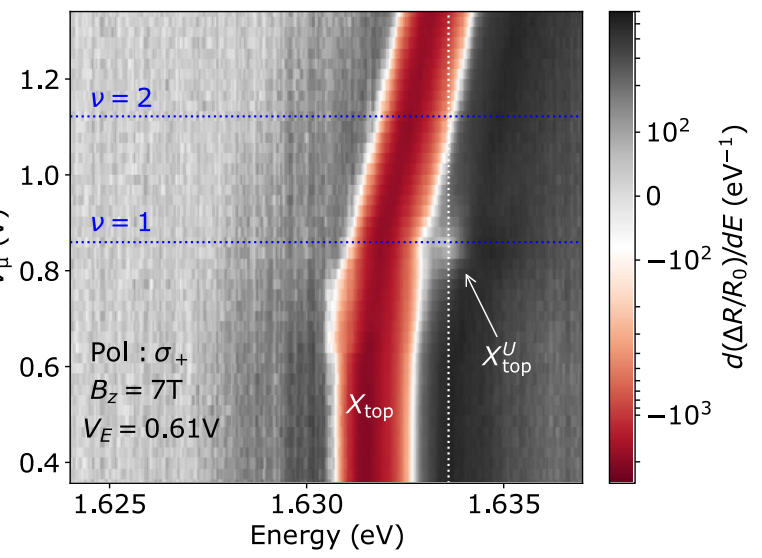

(b)

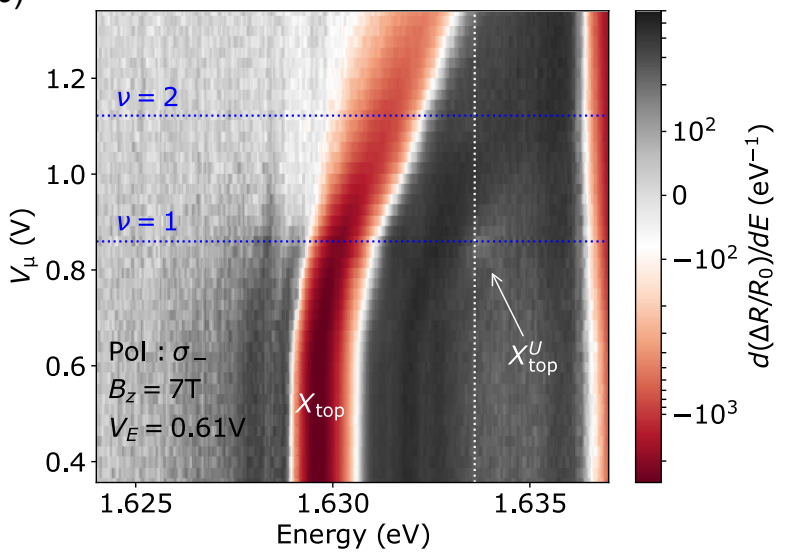

(c)

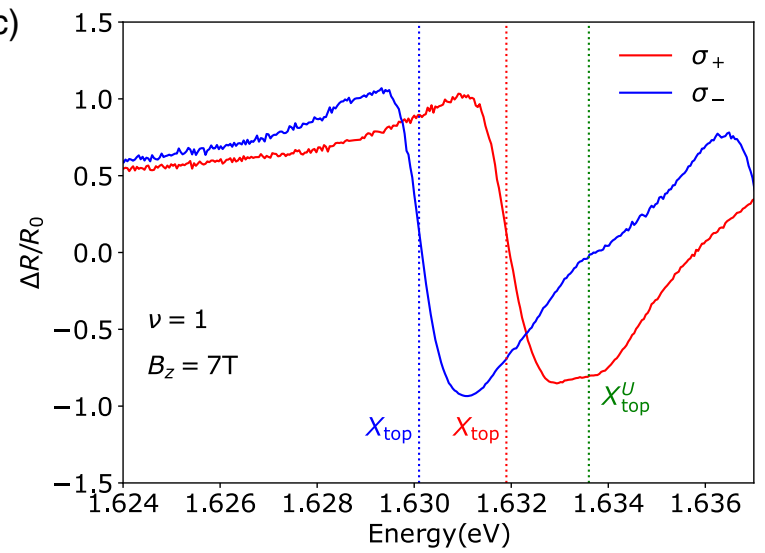

(d)

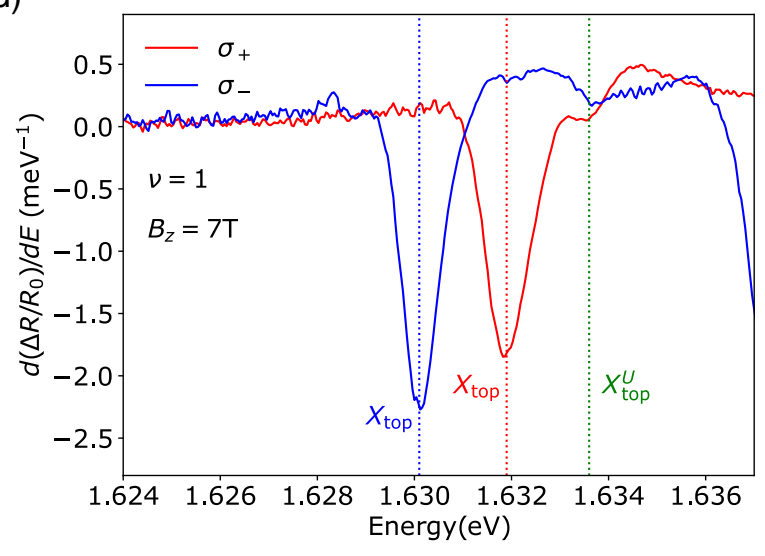

FIG. 5. Exciton and umklapp exciton resonances at $\nu=1$ under magnetic field $B_{z}=7$ T. (a),(b) $V_{\mu}$ dependence of differential reflectance differentiated with respect to energy $E$ in $\sigma_{+}$(a) and $\sigma_{-}$(b) polarization. (c),(d) Line cuts of the reflectance spectrum (c) and its energy differentiation (d) at $\nu=1, V_{E}=0.61 \mathrm{~V}$.

interaction leads to a large energy splitting of longitudinal and transverse exciton polarized branches for $\mathbf{k}=\mathbf{G}_{m}$, umklapp states react only weakly to magnetic fields perpendicular to the sample. This result allows for a unique and unambiguous test for the finite-momentum character of the observed umklapp resonances: While the main peaks acquire a splitting of approximately $4 \mu_{B} B_{z}$, the umklapp peaks exhibit a much smaller splitting. To illustrate this result, we show the full band structure of the exciton for a magnetic field of 7T in Fig. 4(b) (right).

We experimentally confirm the suppression of Zeeman splitting for umklapp states for $B_{z}=7 \mathrm{~T}$. We perform polarization resolved $\Delta R / R_{0}$ measurements and observe the emergence of umklapp states $\left(X_{\text {top }}^{U}\right)$ in both $\sigma_{+}$ [Fig. 5(a)] and $\sigma_{-}$[Fig. 5(b)] polarization around $\nu=1$ where $\left(\nu_{\text {top }}, \nu_{\text {bot }}\right)=(1,0)$. Figures 5(c) and 5(d) show the line cuts of the $\Delta R / R_{0}$ spectrum and its derivative with respect to emission energy at $\nu=1$ where $\left(\nu_{\text {top }}, \nu_{\text {bot }}\right)=$ $(1,0)$. Compared to the large Zeeman splitting of $X_{\text {top }}$ of $\simeq 2 \mathrm{meV}$, the energy splitting of $X_{\text {top }}^{U}$ is much smaller than $1 \mathrm{meV}$. On the one hand, the observation of vanishingly small Zeeman splitting of the high-energy resonances appearing at $\nu=1$ confirms their identification as umklapp peaks. On the other hand, our measurements provide direct evidence for the important role played by long-range electron-hole exchange interaction for high-momentum exciton states.

\section{DISCUSSION}

In the theoretical analysis, we treat the electronic lattice as rigid and neglect dynamical screening effects such as the distortion of the electronic lattice by the impurity through collective excitations of the CI state. Nevertheless, we expect our static potential model to capture the essential physical processes provided that the first umklapp bands appear below the excitation gap of the electronic state. We estimate, however, that this condition is only marginally satisfied in our experiments, and a quantitative agreement with experiments would require the development of a theory of mobile quantum impurities in a strongly interacting Fermi system.

We also remark that our calculations assume a perfect electronic lattice. Strictly speaking, this assumption is not justified, since we find the electrons in the Mott-like CI 
state to be spin unpolarized and the electron-exciton scattering is known to be spin dependent. Consequently, different exciton-electron scattering channels give rise to similar, but not identical, repulsive interactions [11]. We nevertheless expect our calculation of the umklapp resonances to be qualitatively accurate despite the apparently random electron spin orientation in the moiré lattice sites, since it is known from the theory of alloys that band structures could remain robust and without sizable broadening of the electronic states in the $\Gamma$ valley, even in the presence of disorder [40].

Our observation that the $\sigma^{+}$and $\sigma^{-}$umklapp excitons remain nearly degenerate at $B_{z}=7 \mathrm{~T}$ is a direct consequence of strong intervalley exchange coupling, which ensures that finite-momentum excitons are in-plane linearly polarized, with a longitudinal-transverse energy splitting that is approximately an order of magnitude larger than the Zeeman energy of approximately $2 \mathrm{meV}$. This observation unequivocally demonstrates that the exciton resonances that appear at $\nu=1$ originate from finite-momentum excitons and concurrently allow us to access the physics of high-momentum excitons.

We also remark that there are new repulsive polaron resonances and a fine structure of the attractive polaron resonance for filling factors $\nu \geq 3$ (see Supplemental Material S4 [23]). While we currently cannot provide an explanation for these features, we emphasize that even the underlying electronic state for these high electron densities is so far not understood.

We emphasize that previously reported optical spectroscopy of Mott-like CI states reveal signatures of incompressibility, but direct evidence for the presence of periodic ordering of electrons has been elusive. The observation of the umklapp exciton resonance that we report, on the other hand, is a direct consequence of the emergence of a periodic lattice of electrons. Consistent with the observations reported here, we also observe umklapp peaks associated with the formation of Mott-like correlated states when the structure is hole doped. In particular, we expect the umklapp peak to appear even for compressible states with charge order and that it can provide direct evidence for spontaneous breaking of translational invariance due to the formation of a Wigner crystal [41-44], charge density waves, or bubble phases $[45,46]$ in a defect-free monolayer without an external potential. Even though umklapp resonances that emerge due to spin- or valley-independent exciton scattering are insensitive to homogeneous $B_{z}$, we expect a periodic modulation of exciton Zeeman coupling to lead to significant splitting of the associated umklapp bands even in the presence of strong electron-hole exchange interactions. Recently, a particularly exciting class of so-called "moiré magnets" has been proposed, which allow for a controlled realization of magnetic phases and can feature strong periodic magnetic field variations [47]. In such systems, umklapp scattering of excitons may be an invaluable tool to characterize magnetic order optically.

The data that support the findings of this paper are available in the ETH Research Collection [48].

\section{ACKNOWLEDGMENTS}

This work was supported by the Swiss National Science Foundation (SNSF) under Grant No. 200021-178909/1 and the European Research Council (ERC) Advanced Investigator Grant (POLTDES). Y.S. acknowledges support from the Japan Society for the Promotion of Science (JSPS) overseas research fellowships. K. W. and T. T. acknowledge support from the Elemental Strategy Initiative conducted by MEXT, Japan, A3 Foresight by JSPS and CREST (Grant No. JPMJCR15F3), and JST. We furthermore acknowledge support from the Technical University of Munich Institute for Advanced Study, funded by the German Excellence Initiative, the European Union FP7 under Grant Agreement No. 291763, the Deutsche Forschungsgemeinschaft (DFG, German Research Foundation) under Germany's Excellence Strategy-EXC-2111-390814868, the European Unions Horizon 2020 research and innovation program (Grant Agreement No. 85116), and from the DFG Grants No. KN1254/1-1 and No. KN1254/1-2.

Note added.-Umklapp resonances have been recently used to demonstrate the existence of a Wigner crystal of electrons in two different monolayer $\mathrm{MoSe}_{2}$ devices [49]. Umklapp resonances associated with Wigner crystal states have since been observed in a total of four different samples by a team that includes T. S., Y. S., M. K., and A. I.

\section{APPENDIX A: REFLECTANCE MEASUREMENT}

We perform measurements with a cryogenic confocal microscope setup at a temperature of approximately $4 \mathrm{~K}$. For the reflectance measurements, we illuminate the sample with a single-mode fiber-coupled broadband light-emitting diode with a center wavelength of $760 \mathrm{~nm}$ and a bandwidth of $20 \mathrm{~nm}$.

\section{APPENDIX B: UMKLAPP EXCITON ENERGY FOR WEAK EXCITON-ELECTRON INTERACTION LIMIT}

The energy separation between the exciton and the first umklapp scattered exciton in the weak limit of excitonelectron interaction is given by the following expression:

$$
\Delta E_{X^{U}-X}=\frac{\hbar^{2}\left|\mathbf{G}_{1}\right|^{2}}{2 m_{X}}
$$

where $\mathbf{G}_{1}$ is the lattice vector in Fig. 4(c), which is $\left|\mathbf{G}_{1}\right|=4 \pi /\left(\sqrt{3} a_{M}\right), a_{M}$ is the moiré periodicity, and $m_{X}$ 
is the exciton mass. We use electron effective mass $m_{e}^{*}=$ $0.7 m_{e}$ [32] and hole effective mass $m_{h}^{*}=0.6 m_{e}$ [33,34], which gives $m_{X}=m_{e}^{*}+m_{h}^{*}=1.3 m_{e}$, with $m_{e}$ denoting the free electron mass. For $a_{M}=25 \mathrm{~nm}$, we obtain $\Delta E_{X^{U}-X}=2.5 \mathrm{meV}$.

[1] Y. Cao, V. Fatemi, S. Fang, K. Watanabe, T. Taniguchi, E. Kaxiras, and P. Jarillo-Herrero, Unconventional Superconductivity in Magic-Angle Graphene Superlattices, Nature (London) 556, 43 (2018).

[2] Y. Cao, V. Fatemi, A. Demir, S. Fang, S. L. Tomarken, J. Y. Luo, J. D. Sanchez-Yamagishi, K. Watanabe, T. Taniguchi, E. Kaxiras, R. C. Ashoori, and P. Jarillo-Herrero, Correlated Insulator Behaviour at Half-Filling in Magic-Angle Graphene Superlattices, Nature (London) 556, 80 (2018).

[3] M. Yankowitz, S. Chen, H. Polshyn, Y. Zhang, K. Watanabe, T. Taniguchi, D. Graf, A. F. Young, and C. R. Dean, Tuning Superconductivity in Twisted Bilayer Graphene, Science 363, 1059 (2019).

[4] Y. Tang, L. Li, T. Li, Y. Xu, S. Liu, K. Barmak, K. Watanabe, T. Taniguchi, A. H. MacDonald, J. Shan, and K. F. Mak, Simulation of Hubbard Model Physics in $\mathrm{WSe}_{2} / \mathrm{WS}_{2}$ Moiré Superlattices, Nature (London) 579, 353 (2020).

[5] E. C. Regan, D. Wang, C. Jin, M. I. B. Utama, B. Gao, X. Wei, S. Zhao, W. Zhao, Z. Zhang, K. Yumigeta, M. Blei, J. D. Carlström, K. Watanabe, T. Taniguchi, S. Tongay, M. Crommie, A. Zettl, and F. Wang, Mott and Generalized Wigner Crystal States in $\mathrm{WSe}_{2} / \mathrm{WS}_{2}$ Moiré Superlattices, Nature (London) 579, 359 (2020).

[6] Y. Shimazaki, I. Schwartz, K. Watanabe, T. Taniguchi, M. Kroner, and A. Imamolu, Strongly Correlated Electrons and Hybrid Excitons in a Moiré Heterostructure, Nature (London) 580, 472 (2020).

[7] L. Wang, E.-M. Shih, A. Ghiotto, L. Xian, D. A. Rhodes, C. Tan, M. Claassen, D. M. Kennes, Y. Bai, B. Kim, K. Watanabe, T. Taniguchi, X. Zhu, J. Hone, A. Rubio, A. N. Pasupathy, and C. R. Dean, Correlated Electronic Phases in Twisted Bilayer Transition Metal Dichalcogenides, Nat. Mater. 19, 861 (2020).

[8] Y. Xu, S. Liu, D. A. Rhodes, K. Watanabe, T. Taniguchi, J. Hone, V. Elser, K. F. Mak, and J. Shan, Correlated Insulating States at Fractional Fillings of Moiré Superlattices, Nature (London) 587, 214 (2020).

[9] X. Xu, W. Yao, D. Xiao, and T. F. Heinz, Spin and Pseudospins in Layered Transition Metal Dichalcogenides, Nat. Phys. 10, 343 (2014).

[10] G. Wang, A. Chernikov, M. M. Glazov, T. F. Heinz, X. Marie, T. Amand, and B. Urbaszek, Colloquium: Excitons in Atomically Thin Transition Metal Dichalcogenides, Rev. Mod. Phys. 90, 021001 (2018).

[11] C. Fey, P. Schmelcher, A. Imamoglu, and R. Schmidt, Theory of Exciton-Electron Scattering in Atomically Thin Semiconductors, Phys. Rev. B 101, 195417 (2020).

[12] A. Camjayi, K. Haule, V. Dobrosavljević, and G. Kotliar, Coulomb Correlations and the Wigner-Mott Transition, Nat. Phys. 4, 932 (2008).
[13] M. Sidler, P. Back, O. Cotlet, A. Srivastava, T. Fink, M. Kroner, E. Demler, and A. Imamoglu, Fermi PolaronPolaritons in Charge-Tunable Atomically Thin Semiconductors, Nat. Phys. 13, 255 (2017).

[14] D. K. Efimkin and A. H. MacDonald, Many-Body Theory of Trion Absorption Features in Two-Dimensional Semiconductors, Phys. Rev. B 95, 035417 (2017).

[15] K. L. Seyler, P. Rivera, H. Yu, N. P. Wilson, E. L. Ray, D. G. Mandrus, J. Yan, W. Yao, and X. Xu, Signatures of MoiréTrapped Valley Excitons in $\mathrm{MoSe}_{2} / \mathrm{WSe}_{2}$ Heterobilayers, Nature (London) 567, 66 (2019).

[16] K. Tran et al., Evidence for Moiré Excitons in van der Waals Heterostructures, Nature (London) 567, 71 (2019).

[17] C. Jin, E. C. Regan, A. Yan, M. I. B. Utama, D. Wang, S. Zhao, Y. Qin, S. Yang, Z. Zheng, S. Shi, K. Watanabe, T. Taniguchi, S. Tongay, A. Zettl, and F. Wang, Observation of Moiré Excitons in $\mathrm{WSe}_{2} / \mathrm{WS}_{2}$ Heterostructure Superlattices, Nature (London) 567, 76 (2019).

[18] E. M. Alexeev, D. A. Ruiz-Tijerina, M. Danovich, M. J. Hamer, D. J. Terry, P. K. Nayak, S. Ahn, S. Pak, J. Lee, J. I. Sohn, M. R. Molas, M. Koperski, K. Watanabe, T. Taniguchi, K. S. Novoselov, R. V. Gorbachev, H. S. Shin, V. I. Fal'ko, and A. I. Tartakovskii, Resonantly Hybridized Excitons in Moiré Superlattices in van der Waals Heterostructures, Nature (London) 567, 81 (2019).

[19] T. I. Andersen, G. Scuri, A. Sushko, K. De Greve, J. Sung, Y. Zhou, D. S. Wild, R. J. Gelly, H. Heo, D. Bérubé, A. Y. Joe, L. A. Jauregui, K. Watanabe, T. Taniguchi, P. Kim, H. Park, and M. D. Lukin, Excitons in a Reconstructed Moiré Potential in Twisted $\mathrm{WSe}_{2} / \mathrm{WSe}_{2}$ Homobilayers, Nat. Mater. 20, 480 (2021).

[20] H. Yu, G.-B. Liu, J. Tang, X. Xu, and W. Yao, Moiré Excitons: From Programmable Quantum Emitter Arrays to Spin-Orbit-Coupled Artificial Lattices, Sci. Adv. 3, e1701696 (2017).

[21] F. Wu, T. Lovorn, and A. H. MacDonald, Theory of Optical Absorption by Interlayer Excitons in Transition Metal Dichalcogenide Heterobilayers, Phys. Rev. B 97, 035306 (2018).

[22] D. A. Ruiz-Tijerina and V. I. Fal'ko, Interlayer Hybridization and Moiré Superlattice Minibands for Electrons and Excitons in Heterobilayers of Transition-Metal Dichalcogenides, Phys. Rev. B 99, 125424 (2019).

[23] See Supplemental Material at http://link.aps.org/ supplemental/10.1103/PhysRevX.11.021027 for details on extraction of energy separation between exciton and umklapp exciton resonances, extraction of electron filling periodicity, discussion on blueshift of the main repulsive polaron resonance in a Mott state, and additional data of filling dependence of umklapp exciton resonances.

[24] A. Catellani, M. Posternak, A. Baldereschi, and A. J. Freeman, Bulk and Surface Electronic Structure of Hexagonal Boron Nitride, Phys. Rev. B 36, 6105 (1987).

[25] V. O. Özçelik, J. G. Azadani, C. Yang, S. J. Koester, and T. Low, Band Alignment of Two-Dimensional Semiconductors for Designing Heterostructures with Momentum Space Matching, Phys. Rev. B 94, 035125 (2016).

[26] K. He, C. Poole, K. F. Mak, and J. Shan, Experimental Demonstration of Continuous Electronic Structure Tuning 
via Strain in Atomically Thin $\mathrm{MoS}_{2}$, Nano Lett. 13, 2931 (2013).

[27] H. J. Conley, B. Wang, J. I. Ziegler, R. F. Haglund, S. T. Pantelides, and K. I. Bolotin, Bandgap Engineering of Strained Monolayer and Bilayer $\mathrm{MoS}_{2}$, Nano Lett. 13, 3626 (2013).

[28] C. R. Zhu, G. Wang, B. L. Liu, X. Marie, X. F. Qiao, X. Zhang, X. X. Wu, H. Fan, P. H. Tan, T. Amand, and B. Urbaszek, Strain Tuning of Optical Emission Energy and Polarization in Monolayer and Bilayer $\mathrm{MoS}_{2}$, Phys. Rev. B 88, 121301(R) (2013).

[29] H. Yu, G.-B. Liu, P. Gong, X. Xu, and W. Yao, Dirac Cones and Dirac Saddle Points of Bright Excitons in Monolayer Transition Metal Dichalcogenides, Nat. Commun. 5, 3876 (2014).

[30] M. M. Glazov, T. Amand, X. Marie, D. Lagarde, L. Bouet, and B. Urbaszek, Exciton Fine Structure and Spin Decoherence in Monolayers of Transition Metal Dichalcogenides, Phys. Rev. B 89, 201302(R) (2014).

[31] D. Y. Qiu, T. Cao, and S. G. Louie, Nonanalyticity, Valley Quantum Phases, and Lightlike Exciton Dispersion in Monolayer Transition Metal Dichalcogenides: Theory and First-Principles Calculations, Phys. Rev. Lett. 115, 176801 (2015).

[32] S. Larentis, H. C. P. Movva, B. Fallahazad, K. Kim, A. Behroozi, T. Taniguchi, K. Watanabe, S. K. Banerjee, and E. Tutuc, Large Effective Mass and Interaction-Enhanced Zeeman Splitting of K-Valley Electrons in $\mathrm{MoSe}_{2}$, Phys. Rev. B 97, 201407(R) (2018).

[33] Y. Zhang, T.-R. Chang, B. Zhou, Y.-T. Cui, H. Yan, Z. Liu, F. Schmitt, J. Lee, R. Moore, Y. Chen, H. Lin, H.-T. Jeng, S.-K. Mo, Z. Hussain, A. Bansil, and Z.-X. Shen, Direct Observation of the Transition from Indirect to Direct Bandgap in Atomically Thin Epitaxial $\mathrm{MoSe}_{2}$, Nat. Nanotechnol. 9, 111 (2014).

[34] M. Goryca, J. Li, A. V. Stier, T. Taniguchi, K. Watanabe, E. Courtade, S. Shree, C. Robert, B. Urbaszek, X. Marie, and S. A. Crooker, Revealing Exciton Masses and Dielectric Properties of Monolayer Semiconductors with High Magnetic Fields, Nat. Commun. 10, 4172 (2019).

[35] D. Yoshioka and H. Fukuyama, Charge Density Wave State of Two-Dimensional Electrons in Strong Magnetic Fields, J. Phys. Soc. Jpn. 47, 394 (1979).

[36] Y. Li, J. Ludwig, T. Low, A. Chernikov, X. Cui, G. Arefe, Y. D. Kim, A. M. van der Zande, A. Rigosi, H. M. Hill, .H. Kim, J. Hone, Z. Li, D. Smirnov, and
T. F. Heinz, Valley Splitting and Polarization by the Zeeman Effect in Monolayer $\mathrm{MoSe}_{2}$, Phys. Rev. Lett. 113, 266804 (2014).

[37] A. Srivastava, M. Sidler, A. V. Allain, D. S. Lembke, A. Kis, and A. Imamoglu, Valley Zeeman Effect in Elementary Optical Excitations of Monolayer WSe ${ }_{2}$, Nat. Phys. 11, 141 (2015).

[38] G. Aivazian, Z. Gong, A. M. Jones, R.-L. L. Chu, J. Yan, D. G. Mandrus, C. Zhang, D. Cobden, W. Yao, and X. D. $\mathrm{Xu}$, Magnetic Control of Valley Pseudospin in Monolayer $\mathrm{WSe}_{2}$, Nat. Phys. 11, 148 (2015).

[39] D. MacNeill, C. Heikes, K. F. Mak, Z. Anderson, A. Kormányos, V. Zólyomi, J. Park, and D. C. Ralph, Breaking of Valley Degeneracy by Magnetic Field in Monolayer $\mathrm{MoSe}_{2}$, Phys. Rev. Lett. 114, 037401 (2015).

[40] V. Popescu and A. Zunger, Effective Band Structure of Random Alloys, Phys. Rev. Lett. 104, 236403 (2010).

[41] E. Wigner, On the Interaction of Electrons in Metals, Phys. Rev. 46, 1002 (1934).

[42] C. C. Grimes and G. Adams, Evidence for a Liquid-toCrystal Phase Transition in a Classical, Two-Dimensional Sheet of Electrons, Phys. Rev. Lett. 42, 795 (1979).

[43] N. R. Cooper, Theory of Exciton Recombination from the Magnetically Induced Wigner Crystal, Phys. Rev. B 53, 10804 (1996).

[44] J. Knörzer, M. J. A. Schuetz, G. Giedke, D. S. Wild, K. De Greve, R. Schmidt, M. D. Lukin, and J. I. Cirac, Wigner Crystals in Two-Dimensional Transition-Metal Dichalcogenides: Spin Physics and Readout, Phys. Rev. B 101, 125101 (2020).

[45] A. A. Koulakov, M. M. Fogler, and B. I. Shklovskii, Charge Density Wave in Two-Dimensional Electron Liquid in Weak Magnetic Field, Phys. Rev. Lett. 76, 499 (1996).

[46] R. Moessner and J. T. Chalker, Exact Results for Interacting Electrons in High Landau Levels, Phys. Rev. B 54, 5006 (1996).

[47] K. Hejazi, Z.-X. Luo, and L. Balents, Noncollinear Phases in Moiré Magnets, Proc. Natl. Acad. Sci. U.S.A. 117, 10721 (2020).

[48] Y. Shimazaki, ETH Research Collection, https://www .research-collection.ethz.ch/handle/20.500.11850/428029.

[49] T. Smoleński, P. E. Dolgirev, C. Kuhlenkamp, A. Popert, Y. Shimazaki, P. Back, M. Kroner, K. Watanabe, T. Taniguchi, I. Esterlis, E. Demler, and A. Imamoğlu, Observation of Wigner Crystal of Electrons in a Monolayer Semiconductor, arXiv:2010.03078. 